\title{
Bridging cytoskeletal intersections
}

\author{
Elaine Fuchs ${ }^{1}$ and Iakowos Karakesisoglou \\ Howard Hughes Medical Institute, Department of Molecular Genetics and Cell Biology, The University of Chicago, \\ Chicago, Illinois 60637, USA
}

The cells of multicellular organisms come in fascinating shapes and sizes that are nearly as varied as the animals themselves. Cytoarchitecture is responsible for this diversity and also contributes greatly to the multifaceted functions of each cell type. Historically, the three major cytoskeletal networks-actin microfilaments, intermediate filaments (IFs), and microtubules-were viewed as independent entities, and cytoskeletal researchers focused on the most important functions ascribed to each of these networks. From these initial studies, actin microfilaments were found to play pivotal roles in cell polarity and contractile and migratory processes. In contrast, IFs impart intracellular mechanical strength and are consequently especially abundant in tissues such as epidermis and muscle that undergo substantial physical stress. Cytoplasmic microtubules are vital for intracellular trafficking of vesicles, organelles, and proteins, whereas spindle microtubules function in the dynamics of chromosome alignment and segregation during mitosis.

As cell biologists searched for functional differences between cytoskeletal networks and identified proteins specifically aligned with each network, they discovered that agents perturbing one filamentous network often affected the others and that functions ascribed to one network were sometimes also features of another network. In the past $5 \mathrm{yr}$, it has become increasingly clear that multiple cytoskeletal networks cooperate to perform many of their tasks. Facilitating these close encounters are connector proteins that have dual capacities to associate with more than one cytoskeleton, enabling them to integrate old functions and generate new ones. Many of the proteins initially found to associate with microtubules, and hence called microtubule-associated proteins (MAPs), have been found to also associate with actin microfilaments. A number of microtubule-based motor proteins (kinesins and dynein) and actin-based motor proteins (myosins) have now been found to be members of multiprotein, bifunctional motor complexes that can maneuver along multiple cytoskeletal roadways to transport common cargo. One of the biggest surprises in this topsy turvy world of the cytoskeleton has come

${ }^{1}$ Corresponding author.

E-MAIL nliptak@midway.uchicago.edu; FAX (773) 702-0141.

Article and publication are at www.genesdev.org/cgi/doi/10.1101/ gad.861501. from a growing family of cytoskeletal binding proteins called plakins, which were initially identified on the basis of their association between IFs and membrane junctions (for review, see Ruhrberg and Watt 1997; Wiche 1998; Fuchs and Yang 1999; Coulombe et al. 2000). These proteins are encoded by huge genes that give rise to many different isoforms, each with unique functions and with varying capacities to associate with different cytoskeletal systems. We now know that cytoskeletal cross-linker proteins are involved in many cellular processes ranging from vesicle and organelle transport/orientation to mitosis to adhesion and migration. This review focuses on how cytoskeletal bridges are formed, how they function, and how their connections are regulated.

Introducing the plakins as intermediate filament linker proteins: mechanical integrators of cytoplasm and membranes

While the existence of cytoskeletal cross linkers has been predicted for many years, only recently have proteins been identified that have the capacity to physically link two or more cytoskeletal networks. The best characterized of these are the plakins, an emerging family of sequence-related cross-linker proteins that include plectins, the bullous pemphigoid antigen-1 proteins (BPAG1s), ACF7 (referred to as kakapo in lower eukaryotes), desmoplakin, envoplakin, and periplakin. Plakins are enormous (200-700 kD) proteins that anchor cytoskeletal networks to each other and/or to cellular structures such as adhesive junctions. Thus, for example, BPAG1e (the epithelial form of BPAG1) binds not only to IFs but also to proteins in hemidesmosomes, which are robust integrin-mediated cell substratum junctions that anchor stratified epithelia to their underlying basement membrane (Yang et al. 1996). Desmoplakin, however, associates not only with IFs but also with components of desmosomes, which are specialized cadherin-mediated intercellular junctions (Stappenbeck and Green 1992; Kouklis et al. 1994; Smith and Fuchs 1998; Kowalczyk et al. 1999). More restricted in their expression, periplakin and envoplakin cross link desmosomes and IFs to the cornified envelope of terminally differentiating cells of stratified squamous epithelia (Ruhrberg et al. 1996). One of the most versatile and ubiquitously expressed plakins is plectin, which decorates cytoplasmic IF networks and 
also binds to hemidesmosomes and desmosomes, MAPs, $\alpha$-spectrin, actin filaments, and the nuclear envelope (Wiche et al. 1982; Andra et al. 1997, 1998; Rezniczek et al. 1998; Schaapveld et al. 1998). The association of plakins with IFs changes on the phosphorylation state of serine residues in the carboxy-terminal segment of the plakin, suggesting that this interaction may be regulated within cells. As just one of the intriguing examples in this field, the cyclin-dependent kinase cdk1 phosphorylates plectin and instigates its dissociation from vimentin during mitosis (Foisner et al. 1996).

Most plakin genes encode multiple isoforms that are differentially expressed in a tissue-specific manner (Brown et al. 1995; Bernier et al. 1996; Elliott et al. 1997; Fuchs et al. 1999; Lee et al. 2000). A common feature of the majority of plakin isoforms is the presence of a 6001100 amino acid $\alpha$-helical coiled-coil rod domain that serves as the basis for dimerization of two parallely aligned polypeptide chains. The rod domain is often, but not always, flanked by a carboxyl-terminal IF-binding domain (Fig. 1). The amino-terminal domains of plakins are composed of a number of exons that can be mixed and matched through differential splicing. This enables the resulting isoforms to perform functions uniquely tailored to suit the cytoskeletal needs of each specialized cell (Yang et al. 1996; Fuchs and Yang 1999; Andra et al.
1998; Rezniczek et al. 1998; Karakesisoglou et al. 2000; Lee et al. 2000).

Like IFs themselves, IF-linking plakins are especially prominent in tissues such as muscle and epidermis, which are exposed to substantial mechanical stress. The ability to span a network of tough and resilient IFs across membranes provides mechanical strength to these cells and tissues. Testimony to the importance of these connections is that plectin mutations underlie the cause of a rare form of human muscular dystrophy associated with epidermolysis bullosa simplex (EBS; for review, see Fuchs and Cleveland 1998).

\section{Plakins and other proteins that link actin with intermediate filament networks: strengthening cellular frameworks and adhesion and regulating actin dynamics}

Some plakin isoforms can associate with both IF and actin cytoskeletons (Seifert et al. 1992; Yang et al. 1996; Andra et al. 1998; Karakesisoglou et al. 2000). Rather than link keratin IFs to hemidesmosomes as BPAGle can do, two neuronal forms of BPAG1 (BPAG1n1 and BPAG1n2) contain a bona fide actin binding domain (ABD; Brown et al. 1995; Yang et al. 1996). In addition, they retain the capacity to bind simultaneously to the
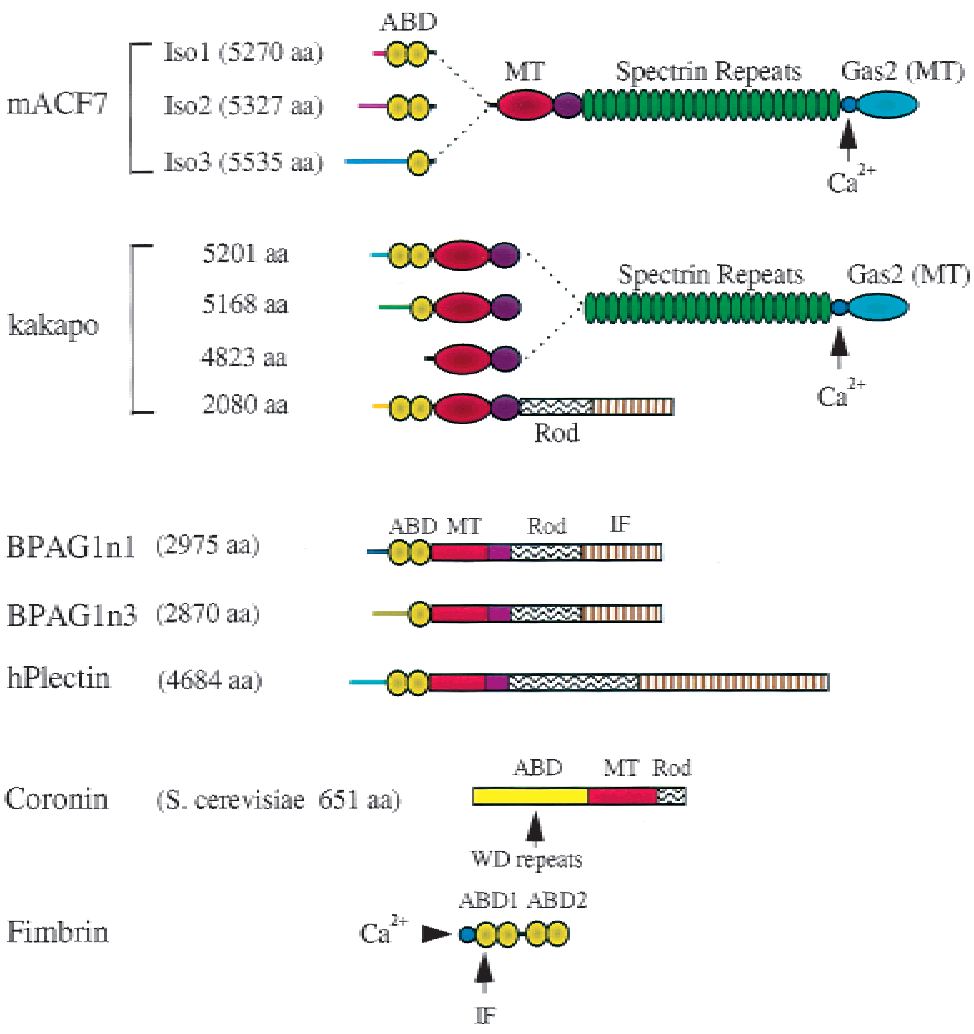

Figure 1. Cytoskeletal linker proteins. Schematic of plakins and cytoskeletal linker proteins. $\mathrm{ABD}$, the actin binding domain, is split into calponin homology $\mathrm{CH} 1$ and $\mathrm{CH} 2$ segments. $\mathrm{CH} 1$ is missing in isoform 3 of both ACF7 and BPAG1n. The kakapo/ACF7/spectrin repeats are similar to those of dystrophin (Strumpf and Volk 1998; Lee et al. 1999). The similarity to calcium-binding EF-hand repeats are noted, as is the Gas2 homology domain (see Strumpf and Volk 1998; Gregory and Brown 1998). Other regions are shaded to denote unique sequences. 
neuronal IF network, perhaps anchoring the neuronal IFs cytoskeleton to the cortical actin filaments lining the axon.

Plectin also associates with actin and IF networks, perhaps stabilizing the actin cytoskeleton by scaffolding it to IFs (Seifert et al. 1992). At hemidesmosomes, however, plectin binds to $\alpha 6 \beta 4$ integrin, which alters its conformation such that it still associates with IFs but not with actin filaments (Fig. 2; Geerts et al. 1999). Conversely, at sites of $\alpha \beta 1$ integrin-rich focal adhesions, plectin associates with the actin cytoskeleton, an interaction that is dependent on plectin's ability to bind phosphaditylinositol-4,5-bisphosphate (PIP2), a signaling molecule and major stimulator of actin polymerization (Fig. 2; Andra et al. 1998).

The biological significance of plectin's association with actin has been revealed in studies using primary fibroblasts cultured from plectin null mice: These cells are more adherent, less motile, and fail to display shortterm rearrangements of actin filaments in response to the Rho family of small GTPases (Andra et al. 1998). It is not yet known whether plectin physically binds and brings the GTPases to the right places or whether it acts by creating the right architectural milieu for GTPase recognition. Irrespective of mechanism, plectin appears to function in actin dynamics and in the rearrangements of actin cytoskeleton that are activated by small GTPase signaling cascades. Considering that this interaction domain is conserved among a number of different plakins, it will be intriguing in the future to probe further into the role of ABD- and PIP2-binding plakins in orchestrating focal adhesion assembly and actin stress fiber formation.

In cultured macrophages, actin filaments and IFs converge at $\beta 1$ integrin-rich focal contacts during cell adhesion and spreading. In these cells, the nonplakin protein fimbrin appears to integrate the actin and vimentin cytoskeletons in an adhesion dependent fashion (Fig. 1; Correia et al. 1999). In contrast to plakins, however, fimbrin possess overlapping binding sites for IFs and f-actin, affording it an opportunity to regulate its associations with the two cytoskeletal networks, either through modifications/changes in fimbrin or the cytoskeletal and/or integrin-associated proteins with which fimbrin interacts. Changes in cytoskeletal binding affinities might explain why focal adhesions in fibroblasts prefer-
HEMIDESMOSOME

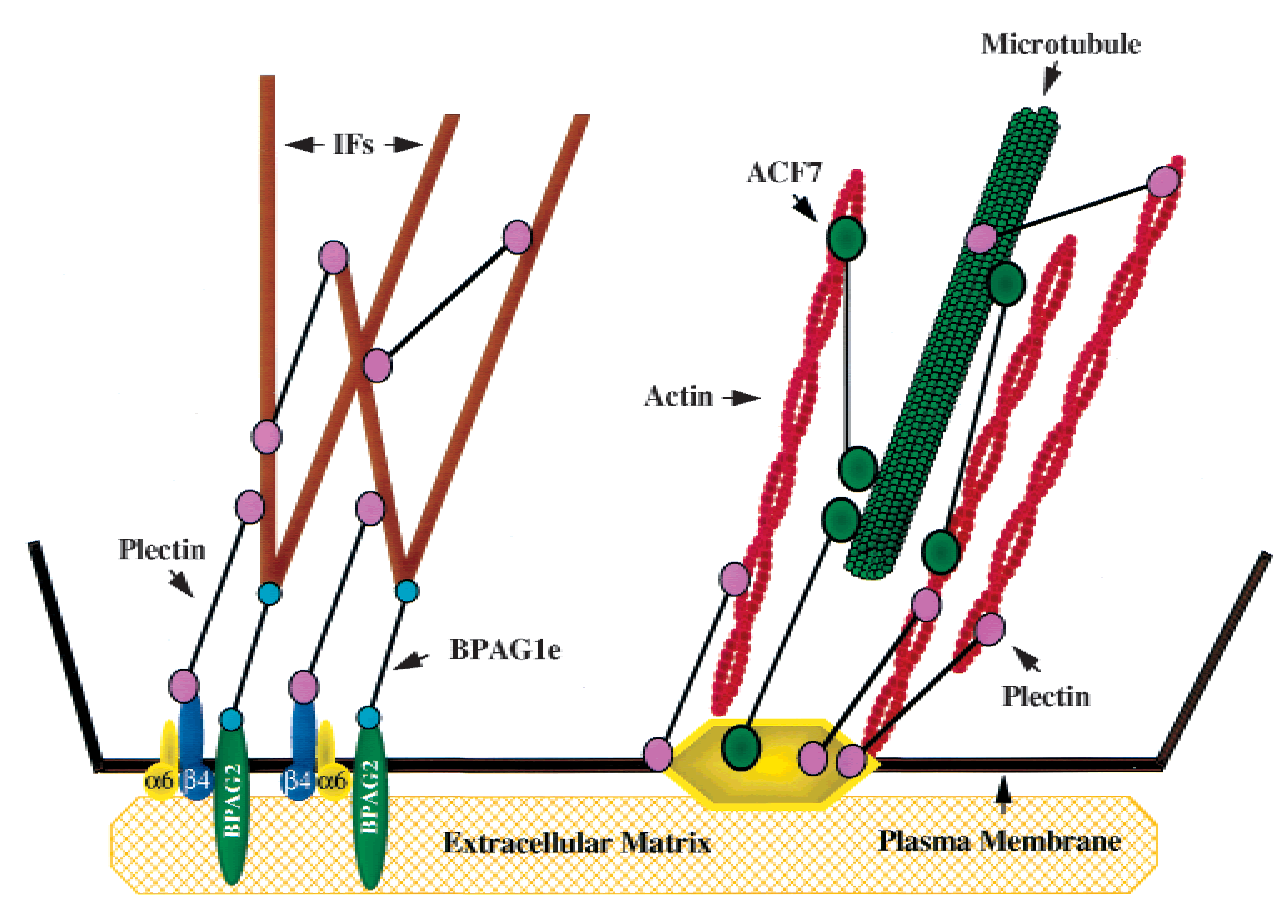

Figure 2. Cytoskeletal crosslinks at integrin-mediated junctions. Schematic of the basal surface of an epidermal cell within mammalian skin. At left is a mammalian hemidesmosomal adherens junction, showing connections to the cytoskeleton, mediated through BPAG1e and plectin - two members of the plakin family. Biochemical studies have shown that BPAG1e associates with BPAG2, a transmembrane protein with an extracellular collagenous domain, as well as with keratin IFs. Plectin associates with $\beta 4$ integrin's atypical 1000-amino acid residue intracellular domain, as well as with keratin IFs and with the cortical actin cytoskeleton. While not demonstrated directly, plectin may also have the capacity to bind to microtubules. At right is a schematic of a focal adhesion site, containing at its core, $\alpha 3 \beta 1$ integrin (depicted here as a yellow hexagon to indicate the fact that there are many other proteins involved at this site). Shown are connections with the actin and microtubule cytoskeletons facilitated by two plakins, kakapo/ACF7 and plectin. Recently, evidence has been accumulating to suggest that microtubules associate with the focal adhesions in mammalian cells as they do in lower eukaryotes (Kaverina et al. 1998). With the exception of plectin and several of the integrin-associated proteins that have no fly counterparts, this type of integrin-mediated junction is likely to be similar in Drosophila. 
entially associate with the actin and not with the IF cytoskeleton.

The amino-terminal segment of those plakins that associate with the actin cytoskeleton harbors an ABD composed of two classical calponin homology $(\mathrm{CH})$ domains similar to those found in spectrin, dystrophin, and fimbrin (Hanein et al. 1998; Keep et al. 1999). Using a procedure that incorporates biochemical, electron density, and related crystal structure data, Matsudaira and colleagues have examined how the ABD in fimbrin binds specifically to f-actin and proposed a model for how fimbrin might cross link a bundle of actin filaments. While such modeling has not yet been performed for the ABDs of plakins, the ability of plakins to dimerize raises the possibility that some plakin isoforms can act as potential actin filament cross-linking agents as well as bridges between actin filaments and IFs.

Adding another dimension to the complexity of plakins' actin filament connections is the existence of several novel isoforms of plakins that differ as a consequence of differential splicing within the conserved set of exons encoding the ABD (Fuchs et al. 1999; Yang et al. 1999; Lee et al. 2000). Interestingly, a novel ABD version contained in the muscle-specific isoform of plectin exhibits significantly higher actin-binding activity than the other ABD splice forms of plectin (Fuchs et al. 1999). These authors have postulated a biological role for this isoform in strengthening the cytoskeletal framework necessary to oppose the intense mechanical forces exerted on tissue such as muscle. In smooth muscle cells, basic calponin (a major actin-, tropomyosin-, and calmodulin-binding protein) may further strengthen actinIF connections: Recent biochemical analyses reveal a potential interaction between this protein and the rod domain of the muscle-specific intermediate filament network composed of desmin (Fujii et al. 2000).

\section{Linking intermediate filament, microtubule and actin cytoskeletons and unveiling a new family of actin-microtubule cross-linking plakins}

Some plakins have the capacity to bridge IFs and microtubules. A direct link was first visualized by immunoelectron microscopy of cultured fibroblasts whose actin cytoskeleton had been disrupted and extracted (Fig. 3A; Svitkina et al. 1996; see also Seifert et al. 1992). Microtubule binding sites have now been identified in several plakins, including a third neuronal isoform of BPAG1 (BPAG1n3) and several ACF7 isoforms (Yang et al. 1999; Leung et al. 1999b; Karakesisoglou et al. 2000) (Fig. 1). BPAG1n3 possesses the classical IF-binding domain but lacks exons encoding the $\mathrm{CH} 1$ part of the ABD (Yang et al. 1996, 1999|. Hence, BPAG1n3 may perform microtubule bundling tasks and/or integrate microtubule and IF cytoskeletons, as hinted by its localization in sensory axons (Fig. 3B). BPAG1n2 differs from BPAG1n3 in that it retains the $\mathrm{ABD}$, raising the possibility that this plakin may serve to connect all three cytoskeletal networks.

In mice, deletions within the BPAG1 gene result in dystonia musculorum, typified by severe neuronal de-
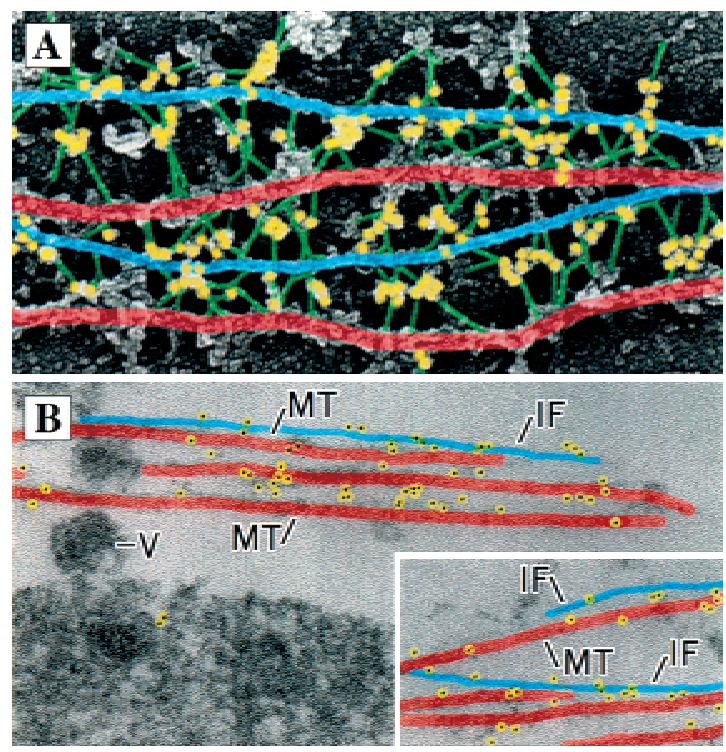

Figure 3. Plakins provide flexible, intracytoplasmic resilience to external stresses by reversibly crosslinking intermediate filaments to other cytoskeletal and membrane sites. (A) Electron micrograph of the residual cytoskeleton of a rat embryo fibroblast after dissolution of actin filaments with gelsolin. Linked to $10-\mathrm{nm}$ gold particles, antibodies against the $500 \mathrm{kD}$ crosslinker protein plectin reveal bridges between microtubules and intermediate filaments. Pseudocolored are: microtubules (red); intermediate filaments assembled from vimentin (blue); plectin (green); gold particles marking plectin (yellow; Svitkina et al. 1996, reproduced with permission of Rockefeller University Press). Image kindly provided by T. Svitkina and G. Borisy. (B) Electron micrograph of the residual cytoskeleton of a mouse dorsal root ganglial axon after extraction of the neuron with nonionic detergent (Yang et al. 1999, reproduced with permission of Elsevier Science). Color coding is as described for $A$, except that the gold particles mark BPAG1n3 antibodies. This plakin has binding sites for microtubules (MT) and intermediate filaments (IF) but lacks the ability to efficiently associate with the actin cytoskeleton (Yang et al. 1999). V, vesicle. Images kindly provided by C. Bauer.

generation throughout the peripheral nervous system by 4-5 wk of age (Guo et al. 1995; Brown et al. 1995). Dorsal root ganglial axons exhibit dramatic disorganization of neuronal IF and microtubule networks accompanied by abnormal axonal myelination and axonal swellings (Dalpe et al. 1998; Eyer et al. 1998, and references therein). Before the discovery of BPAG1n3, this axonal degeneration was attributed to neurofilament (NF) aggregates caused by the failure of NFs to be anchored to the cortical actin cytoskeleton (Yang et al. 1996; see also Leung et al. 1999a). With the discovery of BPAG1n3 and the additional observation that mice lacking both BPAG1 and NFs still display a similar phenotype, NF disorganization can no longer be viewed as the primary culprit that in turn causes disorganization of microtubules in BPAG1-null sensory axons (Yang et al. 1999; see also Eyer et al. 1998). Rather, it now seems more likely that disruption of the microtubule network occurs directly as a consequence of BPAG1 deficiency. Disorgani- 
zation in the microtubule cytoskeleton impedes axonal transport, leading to axonal swellings packed with vesicles and mitochondria that choke the BPAG1-deficient neurons.

The studies with plectin and BPAG1n3 left little doubt that plakins can bind directly to microtubules and that plakins are essential for cytoskeletal integrity and cell survival. The persistence of a phenotype in the BPAG1/ NF-deficient mice further emphasizes that plakins do not always require IFs to function despite the fact that they were first identified as IFAPS, or intermediate filament-associated proteins. In the case of BPAG1n3, immunolocalization studies reveal that the preferred partner of some BPAG1 isoforms is in fact, the microtubule network, making them quintessential MAPs, or microtubule-associated proteins (Yang et al. 1999).

A new twist to the plakin story came from the analysis of genetic mutations in the kakapo gene (also known as short stop), recently shown to encode a plakin (Gregory and Brown 1998; Prokop et al. 1998; Strumpf and Volk 1998). Drosophila is devoid of IF cytoplasmic networks, and hence, kakapo must function through a mechanism independent of this cytoskeleton. However, like most vertebrate plakins, Drosophila kakapo is essential for the function of those cells/tissues that express it, which in this case is for the attachment of epidermis to underlying muscle and for neuromuscular junction formation. In flies, these processes are dependent on PS integrins, related to the $\alpha \beta 1$ integrins in mammals.

In flies, the PS integrins associate with microtubules and with the cortical actin cytoskeleton. Initially, researchers thought that perhaps kakapo functions similarly to BPAGle except that flies utilize microtubules rather than IFs to provide mechanical strength to cellsubstratum junctions. However, recent evidence indicates that ACF7, the more likely mammalian orthologue of Drosophila kakapo (Bernier et al. 1996; Leung et al. $1999 b \mid$, is expressed in epidermal keratinocytes, where it localizes to integrin-rich sites of focal adhesions (Fig. 2; Karakesisoglou et al. 2000). Additional evidence now implicates microtubules in focal adhesion formation in mammalian cells (Kaverina et al. 1998). Thus, kakapo/ ACF7 may have an evolutionarily conserved function in linking the actin and microtubule cytoskeletons to sites of $\alpha \beta 1$-mediated adhesion.

Biochemical studies indicate that the links between kakapo/ACF7, actin, and microtubules are direct. There are at least eight currently known isoforms of this plakin, all of which are unusual in that they lack the characteristic $\alpha$-helical rod and IF-binding domains. In their place is a series of spectrin-like repeats followed by a carboxyl-terminal segment containing putative calcium-regulated EF-hand repeats and a sequence-sharing homology to the growth arrest-specific protein 2, GAS2 (Gregory and Brown 1998; Prokop et al. 1998; Strumpf and Volk 1998; Leung et al. 1999b; Lee et al. 2000; Fig. 1). The classical ABD is present in most, but not all, isoforms, and microtubule-binding domains have been identified in both the carboxyl-terminal domain (Leung et al. $1999 \mathrm{~b}$ ) and in a segment upstream from the spec- trin repeats (Karakesisoglou et al. 2000). Thus, ACF7/ kakapo has the capacity to bundle microtubules as well as to link them to the actin cytoskeleton.

\section{Linking microtubule and actin cytoskeletons: lending direction to cytoskeletal dynamics in cells}

The dramatic degenerative defects seen in the kakapo and BPAG1 mutant animals underscore the importance of actin-microtubule connections to the eukaryotic kingdom, but what are the functions of these connections and why are they so important to cells? The localization of microtubule-actin linking plakins at sites of $\alpha \beta 1$ integrin-mediated adhesion provides a strong clue that one of the roles for these dual cytoskeletal connections resides in cell migration. Substantiating this notion is the recent discovery that certain sensory neurons lacking kakapo exhibit defects in axonal growth cone migration without affecting growth cone morphology and/or navigation (Lee et al. 2000).

$\alpha \beta 1$ integrins, in conjunction with the actin cytoskeleton and its associated myosin motor proteins, orchestrate attachment, spreading, and membrane protrusions in cells. These attachment sites play a pivotal role in migration, where a cell's leading edge transiently adheres through integrins, while the trailing edge detaches from the substratum to move forward (Choquet et al. 1997). Although migration per se does not require microtubules in higher eukaryotes, migration and directionality of movement is impeded if microtubules are depolymerized. Cytoplasmic microtubules align along the axis of cellular movement with their growing ends facing the leading-edge of the cell, and this arrangement appears to be necessary for the normal turnover of cell substratum adhesion sites that must occur behind the protruding front of a moving cell (Kaverina et al. 1998, 2000). In this way, interactions between microtubules and the actin cytoskeleton at sites of focal adhesions seem to function by steering cells and guiding their movement in a directed manner, perhaps selected by an initial chemotactic or wound stimulus.

Using photo-activation marking techniques, Waterman-Storer and Salmon (1997) discovered that the microtubules that are involved in cell migration undergo retrograde flow toward the cell body in an actomyosindependent fashion. During movement, the growing ends of microtubules maintain a distance from the migration front by undergoing a steady net growth of dynamically unstable microtubules toward the leading edge. Waterman-Storer and Salmon propose that polarized actin assembly and retrograde flow at the leading edge of migrating cells is the driving force in creating the gradient of microtubule assembly within the cytoplasm, with net growth at the leading edge and shortening in the cell body (for review, see Waterman-Storer and Salmon 1999). Interestingly, actin microfilaments and microtubules move coordinately during the retrograde flow, providing further evidence that the two are physically linked during the migration process (Fig. 4).

Microtubule-actin cross-linking plakins make good 


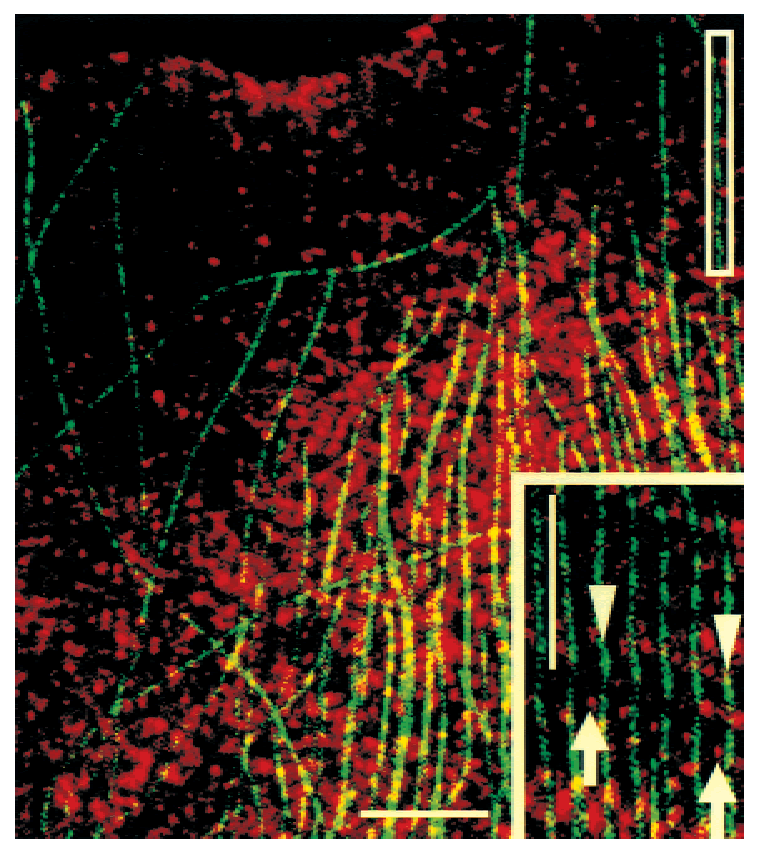

Figure 4. Dual wavelength confocal fluorescent speckle microscopy (FSM) image taken from a time-lapse series of the leading edge lamella of a migrating newt lung epithelial cell that had been coinjected with X-rhodamine actin (red) and Cy-2 tubulin (green). The cell was migrating toward the top of the page, and the images were acquired at 15 -sec intervals. The f-actin meshwork appears to be discontinuously labeled with Cy-2 tubulin along their lengths. These discontinuities in labeling serve as fiduciary marks of the polymer lattices. Inset: dual wavelength kymograph analysis of the region boxed in the main image of MT and f-actin flow in the leading lamella. F-actin speckles (arrow) and a bright speckle along the MT lattice (arrowhead) move coordinately toward the cell center, revealing for the first time the precise coupling of MTs and f-actin during retrograde $\mathrm{f}$-actin flow. This indicates that MTs and f-actin are likely bound by crosslinkers of intermediate to high affinity. Bar $=10$ $\mu \mathrm{m}$. Image courtesy of Wendy C. Salmon and Clare M. Waterman-Storer, The Scripps Research Institute, La Jolla, CA.

candidates for facilitating direct connections between the actin and microtubule networks at the leading edge of moving cells. In mammalian epidermal cells, ACF7/ kakapo is enriched at the periphery of spreading cells, where it often concentrates at the growing ends of microtubules (Fig. 5A,B; Karakesisoglou et al. 2000). ACF7 is often found at sites where actin and microtubules intersect, and in rare cases, where very robust stress fibers occur in epidermal cells, ACF7 preferentially can label these networks (Fig. 5C). Colabeling with antibodies against focal adhesion markers and ACF $7 /$ kakapo reveals that the microtubule tips that are typically decorated by this plakin include the ones that converge at sites of focal adhesions and that intersect with actin stress fibers that associate with these contacts (Fig. 5D,E).

While a role for microtubule-actin connections seems to be firmly entrenched within the proper functioning of cell-substratum adhesions, it is only recently that microtubules have been implicated in the establishment and/or maintenance of cell-cell contacts. WatermanStorer et al. (2000) have now shown that, surprisingly, cells within a sheet of cultured epithelia dissociate on treatment with microtubule depolymerizing agents. Conversely, it was recently discovered that on formation of cadherin-mediated intercellular junctions, the stability of microtubules underlying these junctions is enhanced, thereby revealing an interdependency of the two structures (Chausovsky et al. 2000). Further substantiating a role for microtubule-actin connections in epithelial polarity is the finding that in fly larvae, ectodermally expressed kakapo is required for polarization of the EGF receptor agonist Vein, which is secreted by adjacent myotubes and transmits a signal converting EGF receptor-rich ectodermal cells to tendons (Strumpf and Volk 1998). Another clue stems from the finding that after intercellular adhesion is stimulated, ACF7 and microtubules reorganize in concert and become localized at sites of cell-cell contact (Fig. 5F-J; Karakesisoglou et al. 2000). These findings suggest that ACF7/kakapo plakins may participate in eliciting the changes in cytoskeletal organization necessary to achieve polarity in adhering epithelial cells.

\section{Vesicle trafficking along microtubule-actin highways}

Some actin-microtubule cross-linker proteins are not localized at cell-membrane junctions but, rather, seem to localize to microtubule-actin highways wherever they intersect in the cell cytoplasm. For what purpose? In recent years, it has become increasingly clear that the microtubule and actin cytoskeletons act coordinately to regulate a diverse array of intracellular trafficking, including positioning the nucleus (particularly important in asymmetrically dividing cells), orienting the mitotic spindle with respect to the axis of cell division, and transitioning vesicles and organelles between microtubulebased and actin-based transport systems. Thus, for example, membranous organelles, melanosomes, and even RNAs must shift from microtubule to actin highways as they move from the cell's interior to its periphery. Conversely, endocytosed vesicles must weave through the cortical actin web to the microtubules to reach their interior targets. Such transport relies on the structural reinforcements underlying the two filamentous roadways, as well as the actin and microtubule motors, which must cooperate to transfer cargo from one type of track to another.

Neurons provide excellent examples of cells in which such traffic maneuvering is both routine and spatially well defined. Since the early 1990s it has been known that in neurons an organelle can begin by moving along axonal microtubules until it reaches the growth cone, where it switches to the actin filament network. In this process, the microtubules are used as rails for long-range, rapid transit, whereas actin filament tracks are utilized for bringing the organelle to the tip of a growth conewhich is where the action is (Fig. 6; Kuznetsov et al. 1992).

Located at the crossroads of these two highways is the 

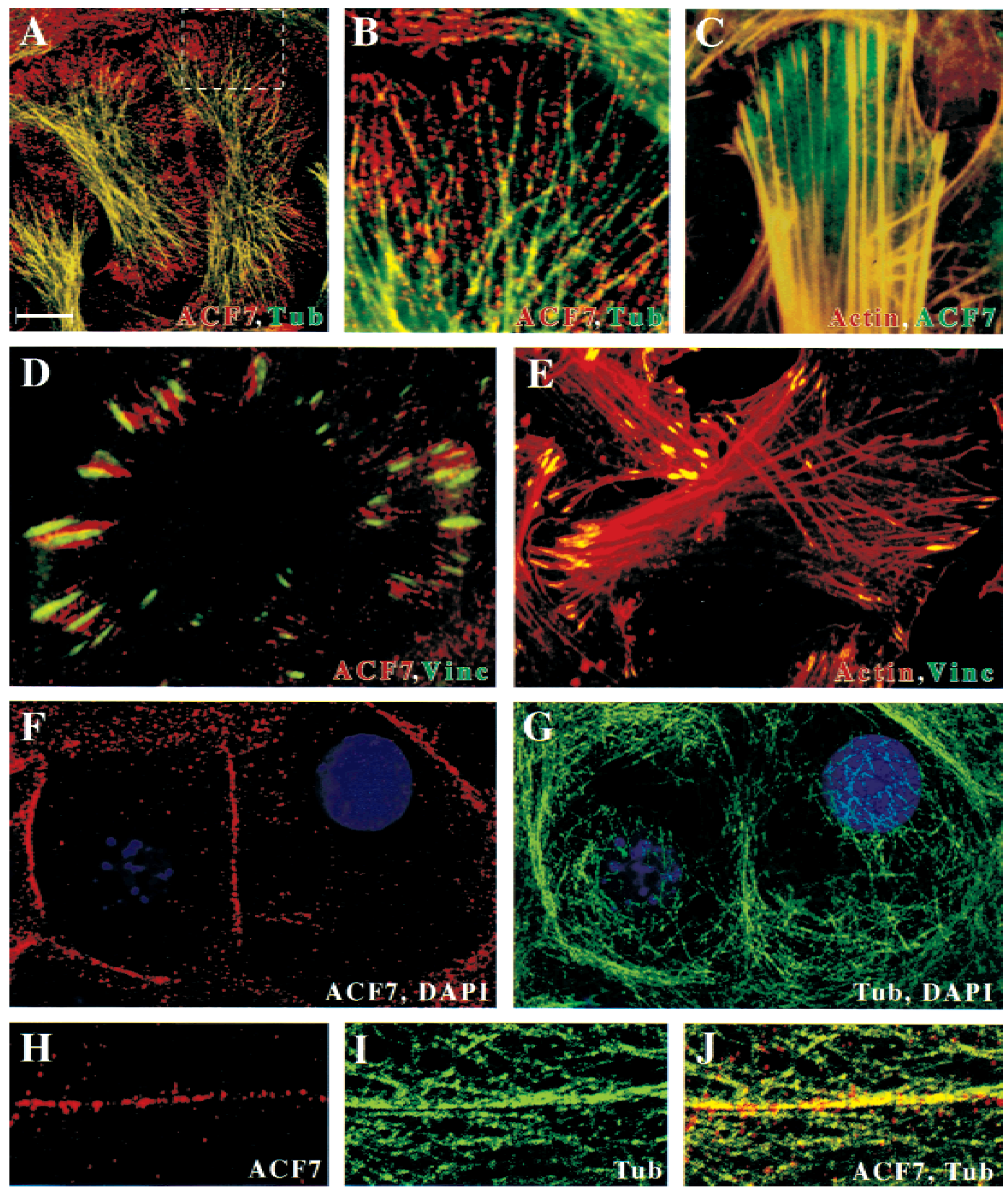

Figure 5. Dynamics of mouse ACF7 in epidermal cells follows cytoskeletal dynamics at sites where microtubules and actin networks interface. Mouse epidermal keratinocytes were cultured on coverslips in medium containing either low calcium $\mid A-E$; $C$ is from Karakesisoglou 2000, reproduced with permission of Rockefeller University Press; $E$ is courtesy of Srikala Raghavan) or high calcium, used to promote cell-cell adhesion $(F-J)$. After washing two times in $0.1 \%$ Triton X-100, $1 \%$ BSA, and $0.1 \%$ gelatin, cells were subjected to immunofluorescence using ACF7, tubulin, and/or actin antibodies as indicated in lower right of each frame. Some cells were counterstained with DAPI (blue). Region in white dotted box of $A$ is shown at higher magnification in $B$. Cell in $F$ and $G$ is same, and frames $H, J$ are higher magnification of a cell-cell border. Bar: $10 \mu \mathrm{m}$ in $A, D, E, F, G ; 3 \mu \mathrm{m}$ in $B, H, I, J ; 5 \mu \mathrm{m}$ in $C$.

nonplakin protein MAP1B, first identified as a microtubule-associated protein (MAP) but recently found to also contain an actin-binding domain (Togel et al. 1998). MAP1B is particularly abundant in the distal axons of neurites, where it could act as a bridge between the axonal microtubule railway and actin cytoskeletal delta of the growth cone. This interaction could be regulated by external and/or intercellular signals, resulting in the phosphorylation of MAP1B, which influences its connection to actin filaments (Togel et al. 1998). Given that mutations in kakapo result in defects in neuronal growth cone migration (Lee et al. 2000), it is also possible that ACF7 also plays a role in transiently stabilizing microtubules through actin connections at the growth cone.

Although most cells do not have quite the flamboyant shape and specialized cytoskeletal needs that neurons 


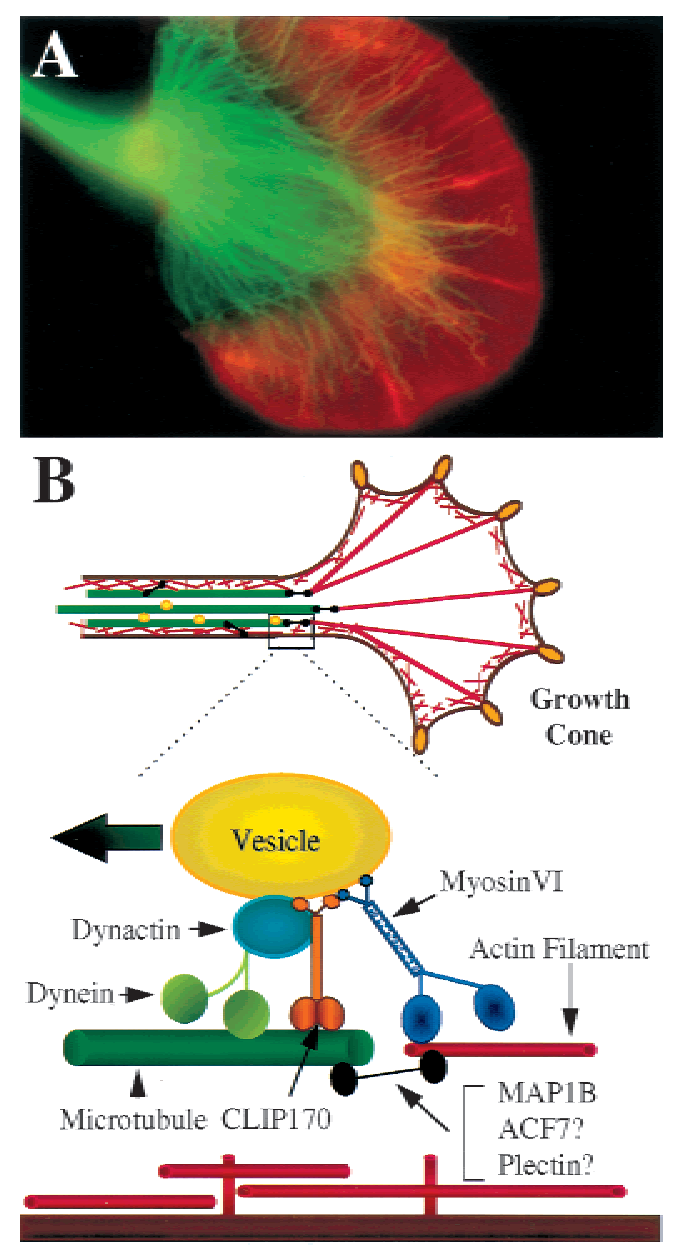

Figure 6. Actin-microtubule connections and their role in directed cytoskeletal dynamics. $(A$; Suter and Forscher 2000. reproduced with permission of Wiley-Liss) Growth cone of a snail neuron, stained with phalloidin (red) to decorate the actin cytoskeleton and antitubulin (green) to label the microtubules. (Courtesy of Paul Forscher, Yale University, New Haven, CT.) (B) Schematic of actin-microtubule connections at the growth cone-axon juncture of neurons. MAP1B and possibly actin-microtubule-linking plakins pave the intersection of axonal microtubules with the growth cone actin framework. At this juncture is a cacophony of traffic, where RNAs and secretory vesicles are transported from the nucleus in the perikarya to the growth cone membrane, while endocytic vesicles are transported in the reverse direction, from the growth cone up to the perikarya. Coordinating these movements are bifunctional motors, consisting of the plus end-directed motors, class V myosins, and kinesins, on the one hand, and the minus end-directed motors, class VI myosins, and dynein, on the other. While the mechanism of neuronal secretory vesicle transport is less clear, the transfer of endocytic cargo is likely to be facilitated by CLIP170 and its associated partners, which can bind not only to the vesicle surface but to class VI myosins and to a subunit of dynactin, the activator of dynein. It seems likely that CLIP-170 acts to coordinate the traffic, binding to a class VI myosin and transferring its cargo to dynactin, which activates dynein and shuttles it up the axonal microtubules.

do, they nevertheless must traffic vesicles and organelles from a microtubule-rich center through a cortical actin- rich periphery. In melanocytes, for instance, pigment granules normally move along a radial array of polarized microtubules, and stimulation of minus-end motors accounts for the aggregation of pigment granules at the cell center. In contrast, however, dispersion of pigment granules throughout the cell is necessary to cause the fish or frog to become darker in skin color, and this involves a switch in motility systems to the actin-myosin network (Rodionov et al. 1998; Rogers and Gelfand 1998). In human skin, melanosomes concentrate at the dendritic tips of the melanocyte, where they are phagocytosed by nearby epidermal cells. The epidermal cells in turn position the melanosomes in the cap over their nucleus to protect against harmful ultraviolet rays.

Genetic dissection of the pathway of melanosome transport was made possible through studies on the dilute mouse and its associated related genetic phenotypes. So named because of its light coat color, the melanocytes of the dilute mutant mouse are defective in melanosome transport and cannot transfer their pigment granules to adjacent skin cells involved in hair production. Underlying this defect is the dilute gene, encoding myosin $\mathrm{Va}$, an actin-based vesicle-transport motor that is likely to function by powering short-range movements that disperse the vesicles delivered by microtubules to actin-rich cellular processes (Brown 1999). The microtubule-based, long-range transport motor, KhcU, binds directly to MyoVa, intimating that actin- and microtubulebased motors act in tandem as a single, bifunctional motor (Huang et al. 1999).

A prerequisite to coordinating actin and microtubule networks is that both cytoskeletal filaments are oriented such that their plus (growing) ends radiate peripherally out toward the plasma membrane. In this way, kinesins, which are plus end-directed microtubule motors, can cooperate with class V myosins, which are plus end-directed actin filament motors (Cheney 1993; Lantz and Miller 1998). Interestingly, minus end-directed motors (actin-based class VI myosins and microtubule-based dynein) are also involved in integrated movements along cytoskeletal roadways (Schliwa 1999; Valetti et al. 1999; Wells et al. 1999), providing a natural means of transporting cargo from the periphery to the interior of cells (for review, see Cramer 2000).

Class V and class VI myosins differ from conventional class II myosins utilized in muscle contraction in that they form dimers rather than polymers /Cheney et al. 1993). The structure of myosin $\mathrm{Va}$ is similar to that of conventional myosins in its amino-terminal domain, which is involved in the binding of ATP, ATPase activity, and the association of the myosin to actin microfilament. Unlike conventional myosins, however, myosin Va has a high affinity for actin in the presence of ATP, enabling it to remain bound to actin filaments for a prolonged time period (Ostap and Pollard 1996; Howard 1997). The tail domain of this myosin is involved in dimerization and binding of organelles and other cargo (Cheney et al. 1993). The dimerization of myosins of the Class V and Class VI myosins provides a mechanism for the molecule to process along an actin filament in a 
hand-over-hand fashion, analogous to kinesin's movement along microtubules (Mehta et al. 1999; see also Berliner et al. 1995; Vale et al. 1996). An added feature of these myosins that cooperate with kinesins is that they possess much longer flexible domains than the class II conventional myosins, and this enables them to take longer steps along an active filament, thereby avoiding the need to move in the helical path along the surface coils of the actin filament (Mehta et al. 1999).

How do the two motors successfully pass their cargo baton to one another? While the precise mechanism remains unknown, evidence suggests that the cargo surfaces themselves play an important role in this process. An appealing model proposes that microtubule-based and actin-based motors both bind to the same cargo as well as to each other to form an integrated motor complex. A key feature of this model would necessitate differential activation of the motors such that during transport down microtubule highways in microtubule-rich cellular domains, the kinesin (or dynein) is activated, but on the switch to actin roads at the cell periphery and/or growth cone, the microtubule motor becomes silent, while the actin-based motors come to life. In this fashion, despite the distinct preference of these motors for their respective cytoskeletons, a seamless transition between microtubule- and actin-based roadways would be possible (Fig. 6). Whether coordinating membrane transport, bidirectional movement of melanophore pigment granules, mitochondrial movement through neuronal cytoplasmic space, or wound closure of plasma membranes in sea urchin eggs, this type of cooperation between microtubule- and actin-based motors is likely to be an important step in facilitating these processes.

While a clear picture is just beginning to emerge for how the roadways and motors of the actin- and microtubule-based cytoskeletons are linked, an equally intriguing problem is how the traffic of vesicles and organelles is governed. In the past few years, researchers have identified conserved microtubule binding proteins that can also bind to endosomes, kinetochores, and other particles in cells. The prototype of this family is human CLIP-170, a microtubule-binding protein that localizes preferentially to the growing ends of microtubules, enabling these proteins to treadmill in partnership with the most dynamic microtubules of the cytoplasm (Perez et al. 1999|. CLIP-170 also interacts with the dynactin complex, a multiprotein activator of the minus end-directed microtubule motor dynein (Vaughan et al. 1999). Perturbation of dynactin function in vivo affects endosome dynamics and trafficking, and dynactin's role appears to be as an obligatory adapter that binds dynein to cargo structures and enhances the processivity of the dynein motor (Valetti et al. 1999). Although one of dynactin's own subunits p150 (Glued) has a microtubule-binding domain, the dynactin association constant for microtubule binding is weak ( 10 $\mu \mathrm{M}$; Waterman-Storer et al. 1995), and hence, CLIP170 may be needed to strengthen the association of the dynactin complex to microtubule plus ends. D-CLIP-190, the likely Drosophila homologue of mammalian CLIP-170, interacts with a myosin VI, lead- ing to the speculation that CLIP-170/D-CLIP-190 acts as a capturing device, establishing initial contact between myosin VI and its associated endosome and then transferring the endosome to the minus end-directed microtubule motor cytoplasmic dynein for transport toward the cell interior (Fig. 6; Pierre et al. 1992; Lantz and Miller 1998).

Whether these proteins also participate in the transfer of cargo in the reverse direction, that is, from the microtubules to the actin cytoskeleton, is presently unknown. While endosome movement is bidirectional, a good place to search for analogous cargo binding proteins that might bind to kinesin and myosin V motors is on the surface of melanosomes or secretory vesicles, which are typically dispersed from the interior to the periphery of the cell. One candidate is kinectin, a kinesin-binding protein that is located on the surface of membrane organelles and seems to be involved in organelle motility /Ong et al. 2000). The kinectin-binding domain on the kinesin tail partially overlaps both its head-binding domain and the Myosin-Va binding domain (Ong et al. 2000). Overall, the multiplicity of interactions between motors, vesicle/ organelle binding proteins, and actin and microtubule cytoskeletons is a reflection of the cacophony of cargo traffic that must occur across complicated and dynamic actin-microtubule crossroads of the cytoskeletal transit system.

\section{Capturing microtubules at cortical sites}

While actin-microtubule connections and molecular motors are important in trafficking cargo, they also have an emerging role in capturing microtubules at specific target sites on the cortical cytoskeleton. A prime example of this occurs during mitosis in budding yeast, where the nucleus and mitotic spindle must align properly along the mother-bud axis to ensure that mother and daughter cells each receive a nucleus. In these cells, the site of the next division is specified at the start of the cell cycle by the position of the bud site. At the start of mitosis, the nucleus rotates and migrates to the bud neck, a process that appears to involve the kinesin Kip3p (HeilChapdelaine et al. 1999). Correct spindle positioning involves a mechanism of search-and-capture, where the dynamic growth properties of cytoplasmic microtubules enable them to search for functional microtubule-capturing centers that are located in the bud cortex (Fig. 7).

Recent genetic evidence implicates Bim1p/EB1, a microtubule-binding protein that is located along microtubules and is particularly concentrated at microtubule distal ends (Lee et al. 2000). Bim1p/EB1 binds to Kar9p, a protein associated with the daughter cell cortex (Miller et al. 1999), and although Bim1p/EB1 is not essential for microtubule capture, it may participate in mediating the transient connection (Lee et al. 2000; Adames and Cooper 2000). On successful encounter, transient binding of microtubules with a specialized cortical complex that includes Kar9p stimulates microtubule depolymerization at the distal end, resulting in shrinkage of the microtubules and dragging of the nucleus to the neck 


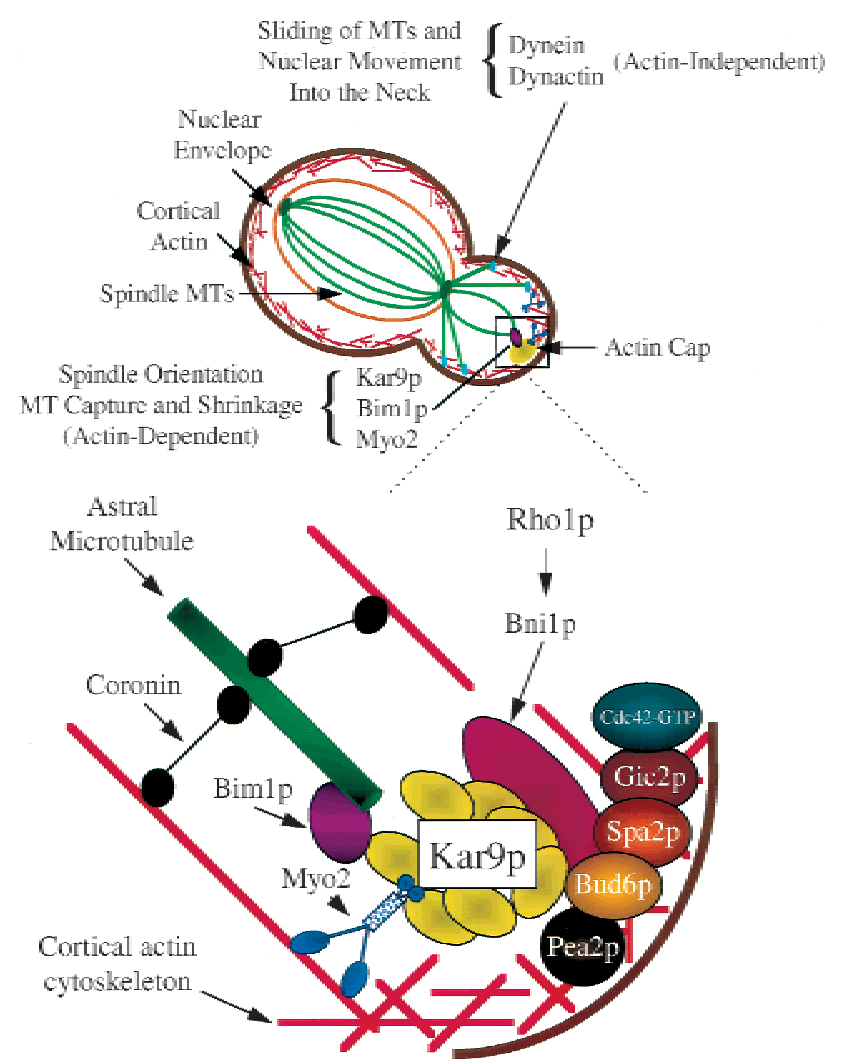

Figure 7. Actin-microtubule connections in budding yeast. Each new bud site dictates the location of a group of proteins that include two Rho GTPase family members, Rholp and its target Bnilp and $\mathrm{Cdc} 42$ and its associated binding protein Gic2p. In addition, Bud6p, Pea2p, Spa2p, and Bnilp appear to serve as a platform for the binding of Kar9p, which may be brought to the bud site as cargo carried by Myo 2. Through its interactions with Bim 1p, Kar9p appears to play a central role in the capture and shrinkage of cytoplasmic microtubules (MT), an actin-dependent process necessary for proper positioning of the nucleus and spindle at the start of mitosis. The dynamic instability that results in rapid depolymerization of microtubules at the bud site is thought to facilitate the movement of nucleus to the bud neck. Subsequent entry of the nucleus into the bud relies on the dynein-dynactin-mediated sliding of microtubules along the inner surface of the bud. This process does not rely upon the actin cytoskeleton per se.

(Adames and Cooper 2000). Capture and shrinkage of microtubule ends can contribute to nuclear movement into the neck, although this is predominantly powered by the sliding of astral microtubules along the bud cortex. This process relies on dynein and dynactin and could be facilitated by virtue of dynactin's ability to indirectly associate with proteins such as Numlp of the actin cortex (Fig. 7; Holleran et al. 1996; see also Efimov and Morris 1998; Heil-Chapdelaine et al. 2000).

Experiments with latrunculin, a potent inhibitor of actin polymerization, illustrate the importance of the actin cytoskeleton in the delivery and anchoring of microtubules to the bud for the Kar9p-dependent capture/shrinkage process (Theesfeld et al. 1999). How is Kar9p trans- ported to the cortex? Myo2, a plus end-directed actinbased motor, has recently been shown to bind to Kar9p (Yin et al. 2000). Myo2 can transport cargo along the cytoplasmic actin cables in yeast, and cables have been shown to be necessary for Kar9p transport to the bud tip (Yin et al. 2000). Thus, Myo2 probably transports Kar9p along actin cables to the cortex to activate the microtubule capture site (Fig. 7). Alternatively, it is possible that Myo2 utilizes binding to Kar9 to contribute to the force necessary to pull cytoplasmic microtubules to the bud tip (Yin et al. 2000).

The mechanism by which the microtubule ends interact with the cortex is not completely understood. Coronin is a protein that may be able to make direct links between these two cytoskeletons (Goode et al. 1999; de Hostos 1999|. In vitro, coronin binds very tightly to actin (Kd $\sim 6 \mathrm{nM}$ ) but only weakly to microtubules (Kd $\sim 20$ $\mu \mathrm{M})$ through a segment that shares some distant homology to MAP1B's microtubule-binding domain (Goode et al. 1999). Crn1 null mutants in budding yeast do not have notable defects in their actin cytoskeleton and do not have mitotic phenotypes similar to Kar9 or dynein mutants; however, they do exhibit subtle microtubule abnormalities (Heil-Chapdelaine et al. 1998). One possibility is that coronin serves as a cytoskeletal linker that perhaps transiently helps to stabilize and/or align microtubules once they are captured through Bimlp and Kar9p interactions.

How does Kar9p get localized to this dynamic, specialized cortical actin attachment site at the bud tip, and how is microtubule capture regulated? While the answers to these key questions remain elusive, genetic and cell biological clues implicate a number of additional players in the process, including Bud6p, Pea2p, Spa2p, Bnilp, and Gic2p (Fig. 7). Actin-binding proteins include Bud6p (Aip3p) and Bnilp (Fujiwara et al. 1999; Lee et al. 1999; Miller et al. 1999). Bnilp is interesting in that its higher eukaryotic cousins, the formins, are thought to be regulators of the actin cytoskeleton (Lee et al. 1999). Bnilp is stimulated by activated Rholp, the yeast homologue of the actin cytoskeletal regulator RhoA, hinting that this small GTPase may control Bnilp activity within the patch (Fujiwara et al. 1999). Gic2p binds to Bud6p, Spa2p, and Cdc42p and seems to act as an adaptor to link Cdc42p to the actin attachment site (Jaquenoud and Peter 2000). These findings hint that the small GTPases orchestrate microtubule-actin dynamics at specialized sites within the bud cortex in a fashion that seems strikingly similar to their role in regulating membrane extensions at the leading edge of migrating cells (Ridley and Hall 1992; Mishima and Nishida 1999).

In fission yeast, as in most higher eukaryotic cells, division is symmetrical, leading to two equal-sized daughter cells. In Saccharomyces pombe, interphase cells utilize both actin and microtubule networks to grow in a polarized fashion at the cell ends and to proceed through cytokinesis. Before mitosis, the nucleus is positioned in the center of the cell by a process that does not appear to require capture of microtubules at the cell 
Cytoskeletal cross-linking proteins

cortex but, rather, a pushing of microtubules against the cell ends (F. Chang, Columbia University Medical School, pers. comm.). Bahler and Pringle (1998) identified a protein Pom $1 p$, which localizes to the cell ends and provides positional information for both growth and division in $S$. pombe. Pom 1 mutants form functioning growth zones and division septa, but following division, cells initiate growth randomly from both ends, rarely switch to bipolar growth, and often misorient their septa, resulting in asymmetric cell division. Interestingly, although pom 1 itself encodes a putative protein kinase, Pom 1p localization to the cell ends requires microtubules and is in the same pathway as Tealp A, which bears resemblance to a group of actin-binding proteins and that localizes to cell ends (Mata and Nurse 1997). Tea2p, a kinesin-like molecule, also localizes to the tips of the cell and to the ends of cytoplasmic microtubules and is required for localization of Tealp to the ends (Browning et al. 2000). Thus, although the mechanism appears to be quite distinct from that of budding yeast, fission yeast seem to position the growth and cytokinesis machineries through interaction with both the actin and microtubule cytoskeletons at specialized cortical attachment sites.

The genetics of orienting the spindle and nucleus and threading them through the yeast bud neck has enabled cell biologists to tease out the molecular details of a very complicated process involving coordination between actin and myosin cytoskeletons. Fortunately, nature has not let all this work go to waste, as the lessons learned from spindle polarization in the yeast bud appear to be operative in higher eukaryotes in a variety of developmental processes ranging from epithelial stratification and tissue morphogenesis to oogenesis to asymmetric cell division and cell fate determination in early embryonic development (Byrne et al. 1994; Skop and White 1998; Gonczy et al. 1999). Many of the same players involved at microtubule-cortical actin intersections in lower eukaryotes have been found at active sites of membrane dynamics and protrusions in higher cells. Thus, at least some elements of the mechanism involving actin-microtubule dynamics in yeast budding are likely to be used broadly in cell polarization events required by higher eukaryotes.

While the precise mechanisms for actin-microtubule function still remain to be elucidated for most of these processes, it has become increasingly clear that throughout the eukaryotic kingdom, various specialized and directed movements require coordination between the cortical actin and microtubule cytoskeletons. Through proteins interacting with cortical actin, microtubule capture can be established. In at least some of these cases, the attachment sites induce dynamic instability and microtubule shrinkage. Microtubule shrinkage can draw microtubule-associated cargo to the membrane, but microtubule depolymerization may also stimulate actomyosin-driven contractility. In this fashion, microtubule depolymerization may participate in locally increasing contractile forces produced by the cell against certain points in the cortex (Elbaum et al. 1999).

\section{Summary}

As we complete the first year of the new millennium, a more integrated view of the cytoskeleton has begun to emerge. While the individual cytoskeletal components retain their identity, these networks rely heavily on one another, employing connections for such diverse functions as cytoskeletal stability, intracellular trasport and trafficking, cytokinesis, cell polarity, and tissue morphogenesis. Key genetic evidence underscores the importance of cytoskeletal connector proteins to cellular architecture and structural integrity in these processes. The recent emergence of linker proteins that bridge between microtubules, actin, and/or IF networks and their accessories, combined with their ancient origins and utmost importance to cell survival and genetic disease, opens a new and fascinating area of cell biology.

Perhaps the most surprising finding to emerge in the past few years is that actin-microtubule associations not only serve to pave the intersections between these cytoskeletal roadways but are also required for cytoskeletal dynamics in a highly regulated fashion. Plectin appears to mediate the cytoskeletal reorganizations that take place on activation by the Rho family of small GTPases, while in yeast budding, the actin-microtubule dynamics appear to be mediated by a constellation of smaller proteins, some of which associate predominantly with actin, others of which interact predominantly with microtubules, and still others that associate with small GTPases. A similar process may occur at the leading edge of migrating mammalian cells, where proteins such as ACF7/kakapo may play a key role. An intriguing parallel at these sites is that the actin-microtubule connection appears to be important in regulating the turnover of focal adhesions, necessary for cellular migration. Actinmicrotubule connections are also likely to be pivotal in regulating epithelial polarity and intercellular adhesion, where adherens junction formation, known to involve actin polymerization and reorganization, has recently been found to occur concomitantly with a reorganization in ACF7 localization and a dramatic change in microtubule localization and stabilization. In all of these scenarios, the actin-microtubule crosslinker proteins appear to participate in conducting cytoskeletal dynamics in a directed fashion. In this "music," they can no longer be considered as mere violinists within the orchestra.

The cytoskeletal connector proteins are Goliaths in both the protein and the isoform world. It is quite likely that up to 20 or even more splice forms exist for some of the plakin genes, which encode proteins of up to $600 \mathrm{kD}$ in size. Judging from the known partnerships of plectin, the complexities are likely to be enormous, and it is quite likely that only the tip of the iceberg has been met so far. The protein surface of these connector proteins remains largely unexplored, and there is plenty of room for as yet unidentified interacting partners. On the basis of their recent functions in cytoskeletal dynamics and cellular movements, some likely candidates include small GTPases and other cytoskeletal regulatory proteins, as well as the docking sites for a variety of mem- 
brane surfaces, including vesicles, mitochondria, golgi, and nuclei. In this regard, we posit that during evolution, genes encoding cytoskeletal linker proteins may have expanded in size and complexity so that they could better coordinate the tailored cytoskeletal dynamics required by specialized cells. Given the enhanced activity and interest in the field of cytoskeletal cross-linking proteins, future research seems certain to continue to reveal new insights into these fascinating regulatory and structural molecules.

\section{References}

Adames, N.R. and Cooper, J.A. 2000. Microtubule interactions with the cell cortex causing nuclear movements in Saccharomyces cerevisiae. J. Cell Biol. 149: 863-874.

Andra, K., Lassmann, H., Bittner, R., Shorny, S., Fassler, R., Propst, F., and Wiche, G. 1997. Targeted inactivation of plectin reveals esential function in maintaining the integrity of skin, muscle, and heart cytoarchitecture. Genes \& Dev. 11: 3143-3156.

Andra, K., Nikolic, B., Stocher, M., Drenckhahn, D., and Wiche, G. 1998. Not just scaffolding: Plectin regulates actin dynamics in cultured cells. Genes \& Dev. 12: 3442-3451.

Bahler, J. and Pringle, J.R. 1998. Pom1p, a fission yeast protein kinase that provides positional information for both polarized growth and cytokinesis. Genes \& Dev. 12: 1356-1370.

Berliner, E., Young, E.C., Anderson, K., Mahtani, H.K., and Gelles, J. 1995. Failure of a single-headed kinesin to track parallel to microtubule protofilaments. Nature 373: 718-721.

Bernier, G., Mathieu, M., De Repentigny, Y., Vidal, S.M., and Kothary, R. 1996. Cloning and characterization of mouse ACF7, a novel member of the dystonin subfamily of actin binding proteins. Genomics 38: 19-29.

Brown, S.S. 1999. Cooperation between microtubule- and actinbased motor proteins. Annu. Rev. Cell Dev. Biol. 15: 63-80.

Brown, A., Bernier, G., Mathieu, M., Rossant, J., and Kothary, R. 1995. The mouse dystonia musculorum gene is a neural isoform of bullous pemphigoid antigen 1. Nat. Genet. 10: 301306.

Browning, H., Hayles, J., Mata, J., Aveline, L., Nurse, P., and McIntosh, J.R. 2000. Tea2p is a kinesin-like protein required to generate polarized growth in fission yeast. I. Cell Biol. 151: 15-28.

Byrne, C., Tainsky, M., and Fuchs, E. 1994. Programming gene expression in developing epidermis. Development 120: 2369 2383.

Chausovsky, A., Bershadsky, A.D., and Borisy, G.G. 2000. Cadherin-mediated regulation of microtubule dynamics. Nat. Cell Biol. 2: 797-804.

Cheney, R.E., O'Shea, M.K., Heuser, J.E., Coelho, M.V., Wolenski, J.S., Espreafico, E.M., Forscher, P., Larson, R.E., and Mooseker, M.S. 1993. Brain myosin-V is a two-headed unconventional myosin with motor activity. Cell 75: 13-23.

Choquet, D., Felsenfeld, D.P., and Sheetz, M.P. 1997. Extracellular matrix rigidity causes strengthening of integrin-cytoskeleton linkages. Cell 88: 39-48.

Correia, I., Chu, D., Chou, Y.H., Goldman, R.D., and Matsudaira, P. 1999. Integrating the actin and vimentin cytoskeletons: Adhesion-dependent formation of fimbrin-vimentin complexes in macrophages. J. Cell Biol. 146: 831-842.

Coulombe, P.A., Bousquet, O., Ma, L., Yamada, S., and Wirtz, D. 2000. The "ins" and "outs" of intermediate filament organization. Trends Cell Biol. 10: 420-428.
Cramer, L.P. 2000. Myosin VI: Roles for a minus end-directed actin motor in cells. J. Cell Biol. 150: 121-126.

Dalpe, G., Leclerc, N., Vallee, A., Messer, A., Mathieu, M., De Repentigny, Y., and Kothary, R. 1998. Dystonin is essential for maintaining neuronal cytoskeleton organization. Mol. Cell Neurosci. 10: 243-257.

de Hostos, E.L. 1999. The coronin family of actin-associated proteins. Trends Cell Biol. 9: 345-350.

Efimov, V.P. and Morris, N.R. 1998. A screen for dynein synthetic lethals in Aspergillus nidulans identifies spindle assembly checkpoint genes and other genes involved in mitosis. Genetics 149: 101-116.

Elbaum, M., Chausovsky, A., Levy, E.T., Shtutman, M., and Bershadsky, A.D. 1999. Microtubule involvement in regulating cell contractility and adhesion-dependent signalling: A possible mechanism for polarization of cell motility. Biochem. Soc. Symp. 65: 147-172.

Elliott, C.E., Becker, B., Oehler, S., Castanon, M.J., Hauptmann, R., and Wiche, G. 1997. Plectin transcript diversity: Identification and tissue distribution of variants with distinct first coding exons and rodless isoforms. Genomics 42: 115-125.

Eyer, J., Cleveland, D.W., Wong, P.C., and Peterson, A.C. 1998. Pathogenesis of two axonopathies does not require axonal neurofilaments. Nature 391: 584-587.

Foisner, R., Malecz, N., Dressel, N., Stadler, C., and Wiche, G. 1996. M-phase-specific phosphorylation and structural rearrangement of the cytoplasmic cross-linking protein plectin involve p34cdc2 kinase. Mol. Biol. Cell 7: 273-288.

Fuchs, E. and Cleveland, D. 1998. A structural scaffolding of intermediate filaments in health and disease. Science 279: 514-519.

Fuchs, E. and Yang, Y. 1999. Crossroads on cytoskeletal highways. Cell 98: 547-550.

Fuchs, P., Zorer, M., Rezniczek, G.A., Spazierer, D., Oehler, S., Castanon, M.J., Hauptmann, R., and Wiche, G. 1999. Unusual $5^{\prime}$ transcript complexity of plectin isoforms: Novel tissue-specific exons modulate actin binding activity. Hum. Mol. Genet. 8: 2461-2472.

Fujii, T., Takagi, H., Arimoto, M., Ootani, H., and Ueeda, T. 2000. Bundle formation of smooth muscle desmin intermediate filaments by calponin and its binding site on the desmin molecule. J. Biochem. (Tokyo) 127: 457-465.

Fujiwara, T., Tanaka, K., Inoue, E., Kikyo, M., and Takai, Y. 1999. Bnilp regulates microtubule-dependent nuclear migration through the actin cytoskeleton in Saccharomyces cerevisiae. Mol. Cell. Biol. 19: 8016-8027.

Geerts, D., Fontao, L., Nievers, M.G., Schaapveld, R.Q., Purkis, P.E., Wheeler, G., Lane, E.B., Leigh, I.M., and Sonnenberg, A. 1999. Binding of integrin $\alpha 6 \beta 4$ to plectin prevents plectin association with F-actin but does not interfere with intermediate filament binding. J. Cell Biol. 147: 417-434.

Gonczy, P., Pichler, S., Kirkham, M., and Hyman A.A. 1999. Cytoplasmic dynein is required for distinct aspects of MTOC positioning, including centrosome separation, in the one cell stage Caenorhabditis elegans embryo. J. Cell Biol. 147: 135-150.

Goode, B.L., Wong, J.J., Butty, A.C., Peter, M., McCormack, A.L., Yates, J.R., Drubin, D.G., and Barnes, G. 1999. Coronin promotes the rapid assembly and cross-linking of actin filaments and may link the actin and microtubule cytoskeletons in yeast. J. Cell Biol. 144: 83-98.

Gregory, S.L. and Brown, N.H. 1998. kakapo, a gene required for adhesion between and within cell layers in Drosophila, encodes a large cytoskeletal linker protein related to plectin and dystrophin. J. Cell Biol. 143: 1271-1282.

Guo, L., Degenstein, L., Dowling, J., Yu, Q.-C., Wollmann, R., 
Perman, B., and Fuchs, E. 1995. Gene targeting of BPAG1: Abnormalities in mechanical strength and cell migration in stratified squamous epithelia and severe neurologic degeneration. Cell 81: 233-243.

Hanein, D., Volkmann, N., Goldsmith, S., Michon, A.M., Lehman, W., Craig, R., DeRosier, D., Almo, S., and Matsudaira, P. 1998. An atomic model of fimbrin binding to F-actin and its implications for filament crosslinking and regulation. Nat. Struct. Biol. 5: 787-792.

Heil-Chapdelaine, R.A., Tran, N.K., and Cooper, J.A. 1998. The role of Saccharomyces cerevisiae coronin in the actin and microtubule cytoskeletons. Curr. Biol. 8: 1281-1284.

Heil-Chapdelaine, R.A., Adames, N.R., and Cooper, J.A. 1999. Formin' the connection between microtubules and the cell cortex. J. Cell Biol. 144: 809-811.

Heil-Chapdelaine, R.A., Tran, N.K., and Cooper, J.A. 2000. Dynein-dependent movements of the mitotic spindle in Saccharomyces cerevisiae do not require filamentous actin. Mol. Biol. Cell 11: 863-872.

Holleran, E.A., Tokito, M.K., Karki, S., and Holzbaur, E.L. 1996. Centractin (ARP1) associates with spectrin revealing a potential mechanism to link dynactin to intracellular organelles. J. Cell Biol. 135: 1815-1829.

Howard, J. 1997. Molecular motors: Structural adaptations to cellular functions. Nature 389: 561-567.

Huang, J.D., Brady, S.T., Richards, B.W., Stenolen, D., Resau, J.H., Copeland, N.G., and Jenkins, N.A. 1999. Direct interaction of microtubule- and actin-based transport motors. Nature 397: 267-270.

Jaquenoud, M. and Peter, M. 2000. Gic2p may link activated $\mathrm{Cdc} 42 \mathrm{p}$ to components involved in actin polarization, including Bnilp and Bud6p (Aip3p). Mol. Cell. Biol. 20: 62446258.

Karakesisoglou, I., Yang, Y., and Fuchs, E. 2000. An epidermal plakin that integrates actin and microtubule networks at cellular junctions. J. Cell Biol. 149: 195-208.

Kaverina, I., Rottner, K., and Small, J.V. 1998. Targeting, capture, and stabilization of microtubules at early focal adhesions. J. Cell Biol. 142: 181-190.

Kaverina, I., Krylyshkina, O., Gimona, M., Beningo, K., Wang, Y.L., and Small, J.V. 2000. Enforced polarisation and locomotion of fibroblasts lacking microtubules. Curr. Biol. 10: 739-742.

Keep, N.H., Norwood, F.L., Moores, C.A., Winder, S.J., and Kendrick-Jones, J. 1999. The $2.0 \AA$ structure of the second calponin homology domain from the actin-binding region of the dystrophin homologue utrophin. J. Mol. Biol. 285: 1257-1264.

Kouklis, P., Hutton, E., and Fuchs, E. 1994. Making the connection: Keratin intermediate filaments and desmosomes proteins. J. Cell Biol. 127: 1049-1060.

Kowalczyk, A.P., Bornslaeger, E.A., Norvell, S.M., Palka, H.L., and Green, K.J. 1999. Desmosomes: Intercellular adhesive junctions specialized for attachment of intermediate filaments. Int. Rev. Cytol. 185: 237-302.

Kuznetsov, S.A., Langford, G.M., and Weiss, D.G. 1992. Actindependent organelle movement in squid axoplasm. Nature 356: $722-725$.

Lantz, V.A. and Miller, K.G. 1998. A class VI unconventional myosin is associated with a homologue of a microtubulebinding protein, cytoplasmic linker protein-170, in neurons and at the posterior pole of Drosophila embryos. J. Cell Biol. 140: 897-910.

Lee, L., Klee, S.K., Evangelista, M., Boone, C., and Pellman, D. 1999. Control of mitotic spindle position by the Saccharomyces cerevisiae formin Bnilp. J. Cell Biol. 144: 947-961.
Lee, S., Harris, K.L., Whitington, P.M., and Kolodziej, P.A. 2000 Short stop is allelic to kakapo, and encodes rod-like cytoskeletal-associated proteins required for axon extension. $J$. Neurosci.20: 1096-1108.

Leung, C.L., Sun, D., and Liem, R.K. 1999a. The intermediate filament protein peripherin is the specific interaction partner of mouse BPAG1-n (dystonin) in neurons. J. Cell Biol. 144: 435-446.

Leung, C.L., Sun, D., Zheng, M., Knowles, D.R., and Liem, R.K. 1999b. Microtubule actin cross-linking factor (MACF): A hybrid of dystonin and dystrophin that can interact with the actin and microtubule cytoskeletons. J. Cell Biol. 147: 12751286.

Mata, J. and Nurse, P. 1997. teal and the microtubular cytoskeleton are important for generating global spatial order within the fission yeast cell. Cell 89: 939-949.

Mehta, A.D., Rock, R.S., Rief, M., Spudich, J.A., Mooseker, M.S., and Cheney, R.E. 1999. Myosin-V is a processive actinbased motor. Nature 400: 590-593.

Miller, R.K., Matheos, D., and Rose, M.D. 1999. The cortical localization of the microtubule orientation protein, Kar9p, is dependent upon actin and proteins required for polarization. J. Cell Biol. 144: 963-975.

Mishima, M. and Nishida, E. 1999. Coronin localizes to leading edges and is involved in cell spreading and lamellipodium extension in vertebrate cells. J. Cell Sci. 112: 2833-2842.

Ong, L.L., Lim, A.P., Er, C.P., Kuznetsov, S., and Yu, H. 2000. Kinectin-kinesin binding domains and their effects on organelle motility. J. Biol. Chem. 275: 32854-32861.

Ostap, E.M. and Pollard, T.D. 1996. Biochemical kinetic characterization of the Acanthamoeba myosin-I ATPase. J. Cell Biol. 132: 1053-1060.

Perez, F., Diamantopoulos, G.S., Stalder, R., and Kreis, T.E. 1999. CLIP-170 highlights growing microtubule ends in vivo. Cell 96: 517-527.

Pierre, P., Scheel, J., Rickard, J.E., and Kreis, T.E. 1992. CLIP-170 links endocytic vesicles to microtubules. Cell 70: 887-900.

Prokop, A., Uhler, J., Roote, J., and Bate, M. 1998. The kakapo mutation affects terminal arborization and central dendritic sprouting of Drosophila motorneurons. J. Cell Biol. 143: 1283-1294.

Rezniczek, G.A., de Pereda, J.M., Reipert, S., and Wiche, G. 1998. Linking integrin $\alpha 6 \beta 4$-based cell adhesion to the in termediate filament cytoskeleton: Direct interaction between the $\beta 4$ subunit and plectin at multiple molecular sites. J. Cell Biol. 141: 209-225.

Ridley, A.J. and Hall, A. 1992. The small GTP-binding protein rho regulates the assembly of focal adhesions and actin stress fibers in response to growth factors. Cell 70: 389-399.

Rodionov, V.I., Hope, A.J., Svitkina, T.M., and Borisy, G.G. 1998. Functional coordination of microtubule-based and actin-based motility in melanophores. Curr. Biol. 8: 165-168.

Rogers, S.L. and Gelfand, V.I. 1998. Myosin cooperates with microtubule motors during organelle transport in melanophores. Curr. Biol. 8: 161-164.

Ruhrberg, C. and Watt, F.M. 1997. The plakin family: Versatile organizers of cytoskeletal architecture. Curr. Opin. Genet. Dev. 7: 392-397.

Ruhrberg, C., Hajibagheri, M.A., Simon, M., Dooley, T.P., and Watt, F.M. 1996. Envoplakin, a novel precursor of the cornified envelope that has homology to desmoplakin. J. Cell Biol. 134: 715-729.

Schaapveld, R.Q., Borradori, L., Geerts, D., van Leusden, M.R., Kuikman, I., Nievers, M.G., Niessen, C.M., Steenbergen, R.D., Snijders, P.J., and Sonnenberg, A. 1998. Hemidesmo- 
some formation is initiated by the $\beta 4$ integrin subunit, requires complex formation of $\beta 4$ and HD1/plectin, and involves a direct interaction between beta 4 and the bullous pemphigoid antigen 180. J. Cell Biol. 142: 271-284.

Schliwa, M. 1999. Myosin steps backwards. Nature 401: 431432.

Seifert, G.J., Lawson, D., and Wiche, G. 1992. Immunolocalization of the intermediate filament-associated protein plectin at focal contacts and actin stress fibers. Eur. J. Cell Biol. 59: $138-147$.

Skop, A.R. and White, J.G. 1998. The dynactin complex is required for cleavage plane specification in early Caenorhabditis elegans embryos. Curr. Biol. 8: 1110-1116.

Smith, E. and Fuchs, E. 1998. Defining the interactions between intermediate filaments and desmosomes. I. Cell Biol. 141: 1229-1241.

Stappenbeck, T.S. and Green, K.J. 1992. The desmoplakin carboxyl terminus coaligns with and specifically disrupts intermediate filament networks when expressed in cultured cells. I. Cell Biol. 116: 1197-1209.

Strumpf, D. and Volk, T. 1998. Kakapo, a novel cytoskeletalassociated protein is essential for the restricted localization of the neuregulin-like factor, vein, at the muscle-tendon junction site. J. Cell Biol. 143: 1259-1270.

Suter, D.M. and Forscher, P. 2000. Substrate-cytoskeletal coupling as a mechanism for the regulation of growth cone motility and guidance. J. Neurobiol. 44: 97-113.

Svitkina, T., Verkhovsky, A., and Borisy, G. 1996. Plectin sidearms mediate interaction of intermediate filaments with microtubules and other components of the cytoskeleton. J. Cell Biol. 135: 991-1007.

Theesfeld, C.L., Irazoqui, J.E., Bloom, K., and Lew, D.J. 1999. The role of actin in spindle orientation changes during the Saccharomyces cerevisiae cell cycle. J. Cell Biol. 146: 10191032.

Togel, M., Wiche, G., and Propst, F. 1998. Novel features of the light chain of microtubule-associated protein MAP1B: Microtubule stabilization, self interaction, actin filament binding, and regulation by the heavy chain. J. Cell Biol. 143: 695707.

Vale, R.D., Funatsu, T., Pierce, D.W., Romberg, L., Harada, Y., and Yanagida, T. 1996. Direct observation of single kinesin molecules moving along microtubules. Nature 380: 451-453.

Valetti, C., Wetzel, D.M., Schrader, M., Hasbani, M.J., Gill, S.R., Kreis, T.E., and Schroer, T.A. 1999. Role of dynactin in endocytic traffic: Effects of dynamitin overexpression and colocalization with CLIP-170. Mol. Biol. Cell 10: 4107-4120.

Vaughan, K.T., Tynan, S.H., Faulkner, N.E., Echeverri, C.J., and Vallee, R.B. 1999. Colocalization of cytoplasmic dynein with dynactin and CLIP-170 at microtubule distal ends. J. Cell Sci. 112: 1437-1447.

Waterman-Storer, C.M. and Salmon, E.D. 1997. Actomyosinbased retrograde flow of microtubules in the lamella of migrating epithelial cells influences microtubule dynamic instability and turnover and is associated with microtubule breakage and treadmilling. J. Cell Biol. 139: 417-434.

- 1999. Positive feedback interactions between microtubule and actin dynamics during cell motility. Curr. Opin. Cell Biol. 11: 61-67.

Waterman-Storer, C.M., Karki, S., and Holzbaur, E.L. 1995. The p150Glued component of the dynactin complex binds to both microtubules and the actin-related protein centractin (Arp-1). Proc. Natl. Acad. Sci. 92: 1634-1638.

Waterman-Storer, C., Duey, D.Y., Weber, K.L., Keech, J., Cheney, R.E., Salmon, E.D., and Bement, W.M. 2000. Microtu- bules remodel actomyosin networks in Xenopus egg extracts via two mechanisms of F-actin transport. I. Cell Biol. 150: 361-376.

Wells, A.L., Lin, A.W., Chen, L.Q., Safer, D., Cain, S.M., Hasson, T., Carragher, B.O., Milligan, R.A., and Sweeney, H.L. 1999. Myosin VI is an actin-based motor that moves backwards. Nature 401: 505-508.

Wiche, G. 1998. Role of plectin in cytoskeleton organization and dynamics. Cell Sci. 111: 2477-2486

Wiche, G., Herrmann, H., Leichtfried, F., and Pytela, R. 1982. Plectin: A high-molecular-weight cytoskeletal polypeptide component that copurifies with intermediate filaments of the vimentin type. Cold Spring Harb. Symp. Quant. Biol. 46: 475-482.

Yang, Y., Dowling, J., Yu, Q.-C., Kouklis, P., Cleveland, D.W., and Fuchs, E. 1996. An essential cytoskeletal linker protein connecting actin microfilaments to intermediate filaments. Cell 86: 655-665.

Yang, Y., Bauer, C., Strasser, G., Wollman, R., Julien, J.P., and Fuchs, E. 1999. Integrators of the cytoskeleton that stabilize microtubules. Cell 98: 229-238.

Yin, H., Pruyne, D., Huffaker, T.C., and Bretscher, A. 2000. Myosin V orientates the mitotic spindle in yeast. Nature 406: $1013-1015$ 


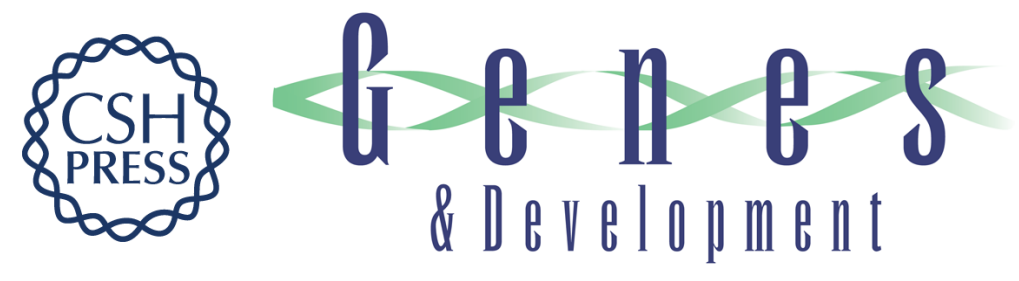

\section{Bridging cytoskeletal intersections}

Elaine Fuchs and lakowos Karakesisoglou

Genes Dev. 2001, 15:

Access the most recent version at doi:10.1101/gad.861501

References This article cites 89 articles, 47 of which can be accessed free at: http://genesdev.cshlp.org/content/15/1/1.full.html\#ref-list-1

License

Email Alerting Receive free email alerts when new articles cite this article - sign up in the box at the top Service right corner of the article or click here.

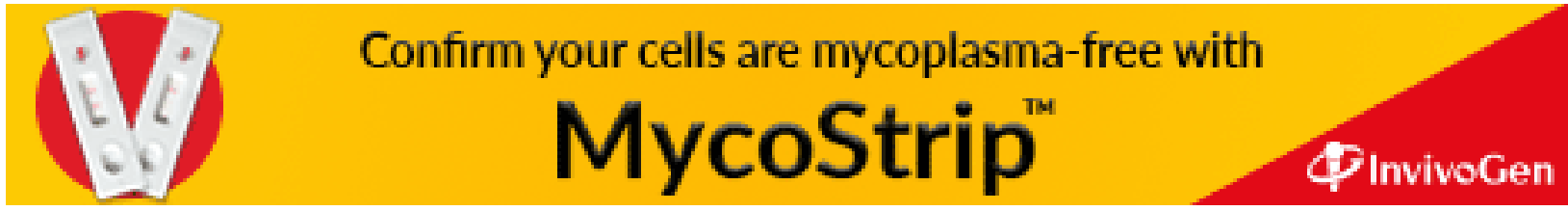

